\title{
Establishment of a nomogram for predicting the surgical difficulty of anterior cervical spine surgery
}

Chengyue $\mathrm{Ji}^{1 \dagger}$, Yuluo Rong ${ }^{1 \dagger}$, Jiaxing Wang ${ }^{1 \dagger}$, Guoyong Yin ${ }^{1}$, Jin Fan ${ }^{1}$, Pengyu Tang ${ }^{1}$, Dongdong Jiang ${ }^{1}$,

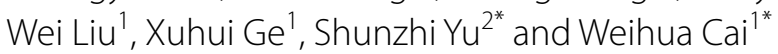

\begin{abstract}
Background: For a long time, surgical difficulty is mainly evaluated based on subjective perception rather than objective indexes. Moreover, the lack of systematic research regarding the evaluation of surgical difficulty potentially has a negative effect in this field. This study was aimed to evaluate the risk factors for the surgical difficulty of anterior cervical spine surgery (ACSS).
\end{abstract}

Methods: This was a retrospective cohort study totaling 291 consecutive patients underwent ACSS from 2012.3 to 2017.8. The surgical difficulty of ACSS was defined by operation time longer than 120 min or intraoperative blood loss equal to or greater than $200 \mathrm{ml}$. Evaluation of risk factors was performed by analyzing the patient's medical records and radiological parameters such as age, sex, BMl, number of operation levels, high signal intensity of spinal cord on T2-weighted images, ossified posterior longitudinal ligament (OPLL), sagittal and coronal cervical circumference, cervical length, spinal canal occupational ratio, coagulation function index and platelet count.

Results: Significant differences were reported between low-difficulty and high-difficulty ACSS groups in terms of age $(p=0.017)$, sex $(p=0.006)$, number of operation levels $(p<0.001)$, high signal intensity $(p<0.001), \mathrm{OPLL}(p<0.001)$ and spinal canal occupational ratio $(p<0.001)$. Multivariate logistic regression analysis revealed that number of operation levels $(O R=5.224,95 \% \mathrm{Cl}=2.125-12.843, \mathrm{p}<0.001)$, high signal intensity of spinal cord $(\mathrm{OR}=4.994,95 \% \mathrm{Cl}=1.636-$ $15.245, \mathrm{p}=0.005), \mathrm{OPLL}(\mathrm{OR}=6.358,95 \% \mathrm{Cl}=1.932-20.931, \mathrm{p}=0.002)$ and the spinal canal occupational ratio $>0.45$ $(\mathrm{OR}=3.988,95 \% \mathrm{Cl}=1.343-11.840, \mathrm{p}=0.013)$ were independently associated with surgical difficulty in ACSS. A nomogram was established and ROC curve gave a $0.906 \mathrm{C}$-index. There was a good calibration curve for difficulty estimation.

Conclusion: This study indicated that the operational level, OPLL, high signal intensity of spinal cord, and spinal canal occupational ratio were independently associated with surgical difficulty and a predictive nomogram can be established using the identified risk factors. Optimal performance was achieved for predicting surgical difficulty of ACSS based on preoperative factors.

Keywords: Surgical difficulty, Anterior cervical spine surgery, Nomogram

*Correspondence: maotoutou@163.com; caiwhspine@sina.com ${ }^{\dagger}$ Chengyue Ji, Yuluo Rong and Jiaxing Wang contributed equally to the manuscript and should be considered co-first authors

${ }^{1}$ Department of Orthopedics, The First Affiliated Hospital of Nanjing Medical University, 300 Guangzhou Road, Nanjing, Jiangsu, China

2 Department of Orthopedics, Shanghai Tenth People's Hospital, Tongji

University School of Medicine, 301 Yanchang Road, Shanghai, China

\section{Background}

Anterior cervical spine surgery (ACSS) is a well-established surgical intervention that has demonstrated longterm clinical benefits in a number of spine conditions such as in the management of degenerative cervical spine 
disease. However, despite its wide application, ACSS is associated with complicated and difficult surgical processes which are often accompanied by high risk and cost. Some of the potential risks that can be disastrous include esophageal injury, epidural hematoma, airway obstruction and vascular injury [1-5]. The surgical difficulty is a pressing concern among surgeons and patients since it highly correlates with the safety and effectiveness of the surgical process. Previously, the evaluation of surgical difficulty has been based on subjective perception rather than objective indexes. In addition, there is a lack of systematic research with regard to the evaluation of surgical difficulty in ACSS. The scientific multi-criteria system is important in decision-making for a surgical process, physician-patient communication, and surgical skills training. This study aimed to evaluate the risk factors for surgical difficulty of anterior cervical spine surgery (ACSS).

\section{Methods}

\section{Study population}

A retrospectively maintained surgical database of 356 consecutive patients who underwent anterior cervical surgery in our center from March 2012 to August 2017 was reviewed. All the patients received written informed consent prior to the surgery. The study was approved by our local institutional ethics committee. The indications for anterior surgery were cervical myelopathy, radiculopathy and ossified posterior longitudinal ligament (OPLL). The exclusion criteria were trauma, tumor, infection, previous cervical surgery and lack of clear preoperative imaging data. Therefore, we analyzed clinical data of 291 patients who underwent anterior cervical spine surgery.

\section{Surgical procedure}

All patients were performed by an experienced spine surgeon (C.W.). The choice of operation was primarily dependent on spinal cord compression. Corpectomy has been effectively used to treat various pathologies including osteophytes, OPLL and nucleus pulposus extending posteriorly to the vertebral body. In this study, discectomy was used if the compression was limited to the disk level. Anterior cervical discectomy and corpectomy were performed with the Smith-Robinson technique. In corpectomy, the optimal titanium mesh cage (DePuy Spine, New Brunswick, New Jersey) was filled with morselized autologous bone and positioned into the defect. In discectomy, PEEK interbody cage (DePuy Spine, New Brunswick, New Jersey) was inserted into the disk space. Anterior cervical locking plates with variable angle screws (DePuy Spine, New Brunswick, New Jersey) were employed in all the patients. Patients were instructed to wear a cervical collar for 6 weeks postoperatively.

\section{Definitions}

The mean time of surgery in this study was $120.3 \mathrm{~min}$ which was chosen as the cut-off. Operation time longer than $120 \mathrm{~min}$ or intraoperative blood loss equal to or greater than $200 \mathrm{ml}$ was considered as a high-difficult surgery. Otherwise, it was defined as low-difficulty surgery. Examined radiological parameters included high signal intensity of spinal cord on T2-weighted images, OPLL, sagittal cervical circumference, coronal cervical circumference, cervical length, anteroposterior diameter of the spinal canal, the shortest diameter of the spinal canal, and spinal canal occupational ratio. Sagittal and coronal cervical circumferences were defined as the distance between the intersection of the line parallel to the endplate through the midpoint of $\mathrm{C} 5$ and soft tissue shadow (Fig. 1). Cervical length was measured as the distance between the sternum and gnathion on the lateral radiograph (Fig. 1a). The anteroposterior and shortest diameter of the spinal canal were measured at the most compressed level using standard picture archiving and communication system (PACS). Anteroposterior diameter was the distance between the posterior edge of the vertebral body and spinal canal while the shortest diameter was defined as the distance between the posterior edge of the compression and spinal canal. The following formula was used to calculate the spinal canal occupational ratio: spinal canal occupational ratio $=$ (anteroposterior diameter-shortest diameter)/anteroposterior diameter (Fig. 2).

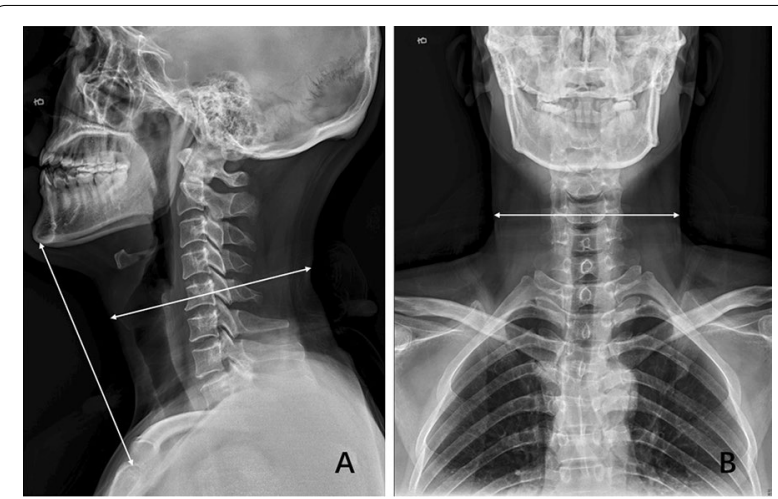

Fig. 1 Sagittal radiograph showing that the cervical length was measured as the distance between the sternum and gnathion. Sagittal and coronal cervical circumferences were defined as the distance between the intersection of the line parallel to the endplate through the midpoint of $\mathrm{C} 5$ and soft tissue shadow 


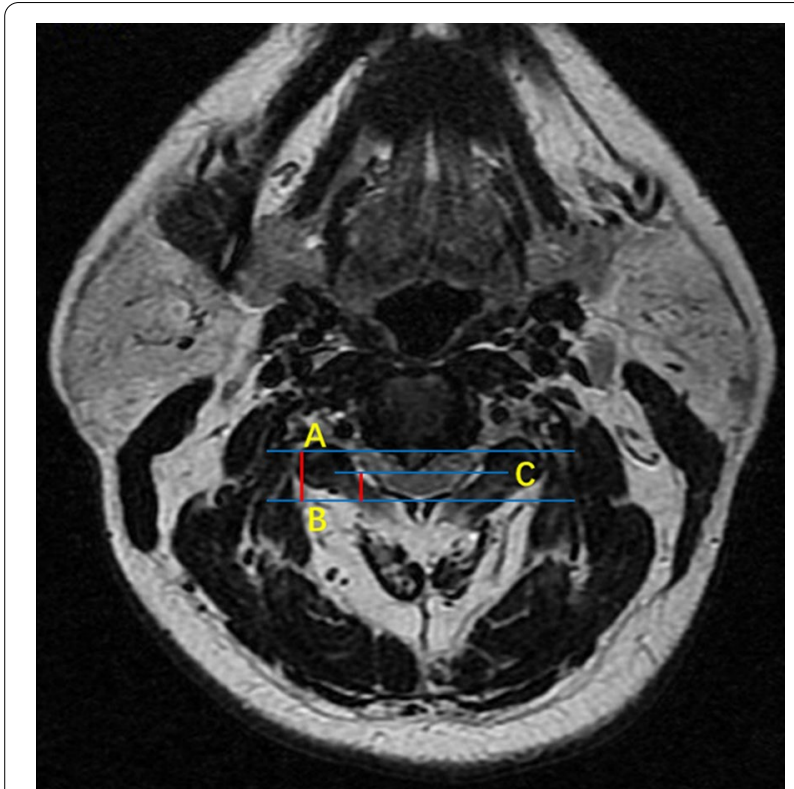

Fig. 2 At the most compressed level, the anteroposterior diameter was defined as the distance between the posterior edge of vertebral body and spinal canal $(\mathbf{a}, \mathbf{b})$. The shortest diameter was the distance between the posterior edge of compression and spinal canal $(\mathbf{b}, \mathbf{c})$. Spinal canal occupational ratio $=$ (anteroposterior diameter-shortest diameter) / anteroposterior diameter

\section{Statistical analysis}

SPSS, version 23.0 and R, version 3.6.1 were used to perform all the data analysis. Continuous variables were expressed as mean \pm standard deviation. Comparisons between the high-difficulty group and low-difficulty group were performed using Student's t-test, MannWhitney $U$ test, $\chi^{2}$ test. Potential risk factors were identified by multivariate logistic regression analysis ( $p$ values $<0.05)$. The predictive value of the multivariate logistic regression was visualized by the receiver operating characteristics (ROC) curves. A nomogram was established using the rms package of $\mathrm{R}$, version 3.6.1. The concordance index ( $C$ index) and a calibration curve were used to determine predictive accuracy. A $P$ value of less than 0.05 was considered statistically significant.

\section{Results}

\section{Patient demographics and clinical characteristics}

Detailed participants' demographics and clinical characteristics in our study are given in Table1. All patients were divided into high-difficulty group $(n=140,48.1 \%)$ and low-difficulty group $(\mathrm{n}=151,51.9 \%)$. Among the 291 patients, $180(61.8 \%)$ were male while $111(38.2 \%)$ were female, with a mean age of 52.3 (range 18-76). The mean surgical time was $120.3 \mathrm{~min}(60-250 \mathrm{~min})$ and
Table 1 Comparison of the low-difficulty and high-difficulty groups

\begin{tabular}{|c|c|c|c|}
\hline Factor & $\begin{array}{l}\text { Low-difficulty } \\
\text { group }(N=151)\end{array}$ & $\begin{array}{l}\text { High-difficulty } \\
\text { group }(N=140)\end{array}$ & $P$ \\
\hline Age*(years) $^{*}$ & $50.99 \pm 10.60$ & $53.84 \pm 9.62$ & 0.017 \\
\hline Sex (male: female) & $82: 69$ & $98: 42$ & 0.006 \\
\hline BMl & $24.15 \pm 3.07$ & $24.74 \pm 2.82$ & 0.154 \\
\hline \multicolumn{4}{|c|}{ Number of operation levels* } \\
\hline 1 & 86 & 7 & \\
\hline 2 & 56 & 77 & \\
\hline 3 & 9 & 53 & \\
\hline 4 & 0 & 3 & $<0.001$ \\
\hline \multicolumn{4}{|c|}{ High signal intensity of spinal cord* } \\
\hline Yes & 52 & 96 & $<0.001$ \\
\hline No & 94 & 49 & \\
\hline \multicolumn{4}{|l|}{ OPLL* } \\
\hline Yes & 17 & 74 & $<0.001$ \\
\hline No & 136 & 64 & \\
\hline $\begin{array}{l}\text { Sagittal Cervical Circum- } \\
\text { ference }\end{array}$ & $14.36 \pm 1.99$ & $14.86 \pm 2.14$ & 0.053 \\
\hline $\begin{array}{l}\text { Coronal Cervical Circum- } \\
\text { ference }\end{array}$ & $12.41 \pm 1.41$ & $12.74 \pm 1.38$ & 0.057 \\
\hline Cervical Length & $14.56 \pm 2.10$ & $14.63 \pm 1.83$ & 0.819 \\
\hline Occupying Ratio* & $0.37 \pm 0.15$ & $0.49 \pm 0.11$ & $<0.001$ \\
\hline PT & $11.70 \pm 0.66$ & $11.74 \pm 0.71$ & 0.708 \\
\hline INR & $1.01 \pm 0.05$ & $1.02 \pm 0.06$ & 0.660 \\
\hline APTT & $28.06 \pm 2.50$ & $27.90 \pm 2.71$ & 0.684 \\
\hline $\mathrm{FIB}$ & $2.64 \pm 0.98$ & $2.44 \pm 0.73$ & 0.130 \\
\hline TT & $18.26 \pm 1.03$ & $18.50 \pm 1.46$ & 0.189 \\
\hline DD2 & $0.32 \pm 0.37$ & $0.33 \pm 0.59$ & 0.924 \\
\hline PLT & $213.82 \pm 79.45$ & $201.49 \pm 51.96$ & 0.210 \\
\hline
\end{tabular}

$B M I$ body mass index, OPLL ossification of the posterior longitudinal ligament *Statistically significant difference

mean blood loss was $33.1 \mathrm{ml}(5-400 \mathrm{ml})$. In the low-difficulty group, one patient had cerebrospinal fluid leakage and two patients had dysphagia. Whereas in the highdifficulty group, the main complications reported were cerebrospinal fluid leakage in three patients, epidural hematoma in one patient and dysphagia in four patients.

\section{Establishment of a nomogram for predicting surgical difficulty}

Comparisons between the low-difficulty and high difficulty groups showed that age $(p=0.017)$, sex $(p=0.006)$, number of operation levels $(p<0.001)$, high signal intensity $(p<0.001)$, OPLL $(p<0.001)$ and spinal canal occupational ratio $(p<0.001)$ significantly correlated with surgical difficulty. The ROC curves further showed that the best thresholds for age and spinal 
canal occupational ratio were 55 (sensitivity: 53.2\%; specificity: $68.9 \%$ ) and 0.45 (sensitivity: $74.2 \%$; specificity: $70.5 \%$ ). Multivariate logistic regression analysis revealed that number of operation levels $(\mathrm{OR}=5.224$, $95 \% \mathrm{CI}=2.125-12.843, \mathrm{p}<0.001)$, high signal intensity $(\mathrm{OR}=4.994,95 \% \mathrm{CI}=1.636-15.245, \mathrm{p}=0.005), \mathrm{OPLL}$ $(\mathrm{OR}=6.358, \quad 95 \% \mathrm{CI}=1.932-20.931, \mathrm{p}=0.002)$ and the spinal canal occupational ratio $>0.45(\mathrm{OR}=3.988$, $95 \% \mathrm{CI}=1.343-11.840, \mathrm{p}=0.013$ ) were independent risk factors for surgical difficulty of anterior cervical surgery (Table 2). Based on these results, number of operation levels, high signal intensity of spinal cord, OPLL and spinal canal occupational ratio can be used to establish a nomogram for use in predicting surgical difficulty (Fig. 3).

Table 2 Multivariate logistic regression analysis

\begin{tabular}{llr}
\hline Factor & Odds ratio $(95 \% \mathrm{Cl})$ & $P$ \\
\hline Age $>55$ & $1.507(0.505-4.494)$ & 0.462 \\
Sex & $0.708(0.224-2.241)$ & 0.557 \\
Number of operation levels & $5.224(2.125-12.843)$ & $<0.001$ \\
High signal intensity & $4.994(1.636-15.245)$ & 0.005 \\
OPLL & $6.358(1.932-20.931)$ & 0.002 \\
Occupying Ratio $>0.45$ & $3.988(1.343-11.840)$ & 0.013 \\
\hline
\end{tabular}

$\mathrm{Cl}$ indicates confidence interval

OPLL ossification of the posterior longitudinal ligament

\section{Evaluation of prediction nomogram}

The ROC curve for the nomogram model showed that the C-index was 0.906, which was an indication of a robust discriminative ability (Fig. 4a). The calibration curve demonstrated that the probability of high-difficulty predicted by the nomogram model agreed well with actual practice (Fig. 4b).

\section{Discussion}

As the population ages, the incidence of degenerative cervical disease is continuously increasing, which is the foremost cause of neurological deficit among adults worldwide $[6,7]$. There is a paradigm shift in the treatment of degenerative cervical disease from posterior to anterior decompression, due to the direct removal of spinal cord compressions, such as disc herniation, osteophytes, and OPLL [8-10]. However, the anterior approach is associated with potential risks that can cause life-threatening complications in vulnerable organs anterior to the vertebral body, such as trachea, esophagus and blood vessels [3]. Therefore, cervical spine surgery is associated with higher surgical difficulty than lumbar surgery hence the need to evaluate surgical difficulty and the associated risk factors for optimal performance in ACSS. From the perspective of developing surgical protocols, assessment of surgical procedure and surgical skill training, a classification system determining the difficulty level is extremely important. Moreover, adequate physician-patient communication is necessary for patients with high surgical difficulty.

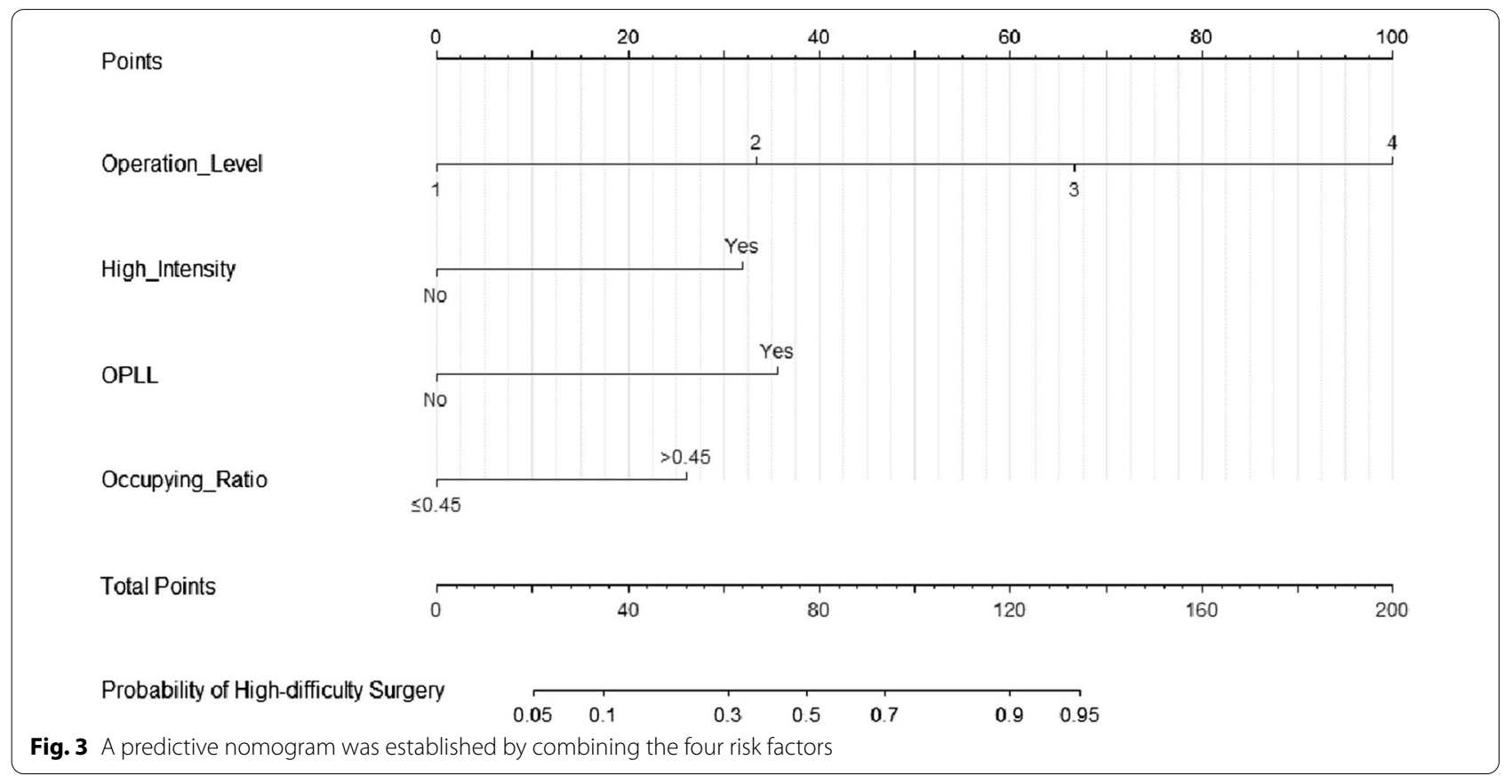



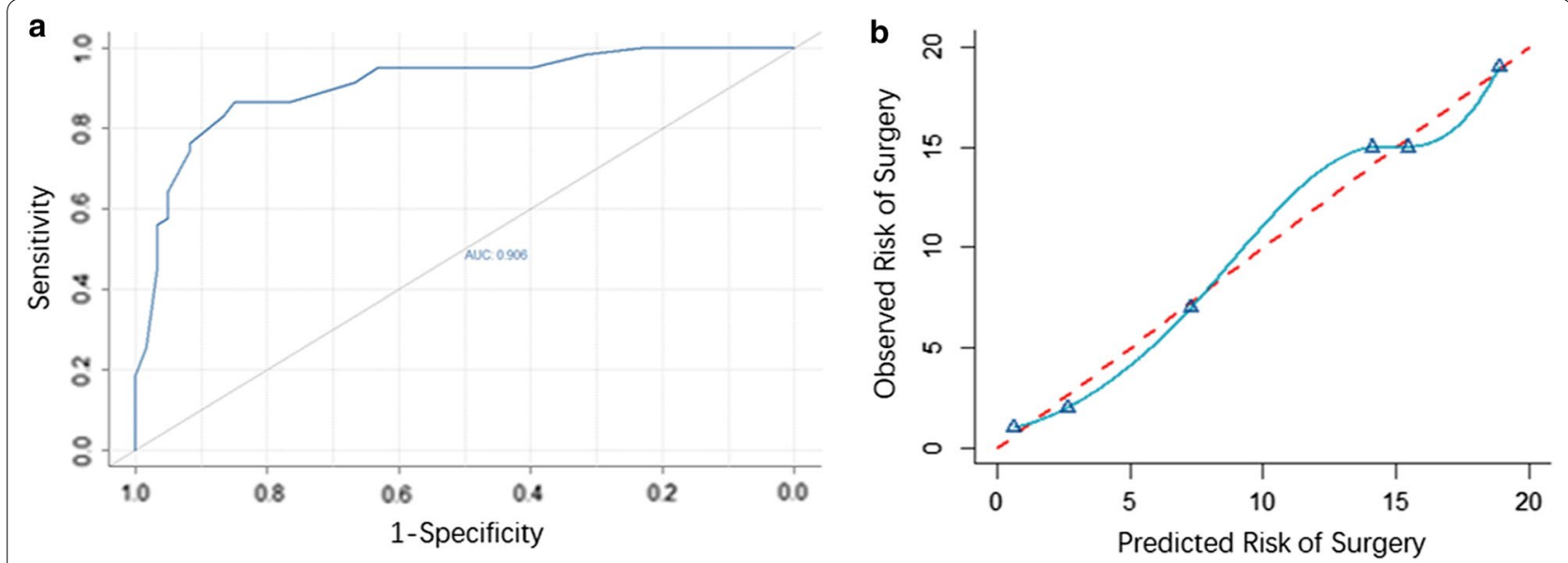

Fig. 4 The ROC curve (a) demonstrated that the C-index was 0.906. b The calibration curve showed that the probability of high-difficulty predicted by the nomogram agreed well with actual practice

Researchers have established several scoring systems for assessing the surgical difficulty. Natkaniec et al. [11] demonstrated that gender, tumor size, and localization are some of the parameters associated with the level of the surgical difficulty of the laparoscopic lateral transperitoneal adrenalectomy. Hasegawa et al. [12] established a novel model for the prediction of surgical difficulty in laparoscopic liver resection and included factors such as the extension of resection, location of tumor, obesity and platelet count. However, a preoperative scoring system evaluating the surgical difficulty of ACSS has not been reported. Therefore, due to lack of a gold standard to estimate for surgical difficulty, surrogate indicators are often used to indirectly reflect the difficulty and these include operative time, intraoperative blood loss and incidence of complication [11-14]. High-difficulty ACSS has been correlated with longer operative time and more intraoperative bleeding.

In this study, a number of parameters such as age, sex, BMI, number of operation levels, high signal intensity of spinal cord on T2-weighted images, OPLL, sagittal cervical circumference, coronal cervical circumference, cervical length, spinal canal occupational ratio, and coagulation function index were evaluated to identify risk factors for high-difficulty ACSS. Previous studies confirm that the elderly, obese patients and male gender are associated with longer operative time [11, 12, 15]. However, this study reported that there is no significant difference in terms of age, sex, and BMI between low-difficulty and high-difficulty groups of ACSS. Coagulation function index and platelet count are associated with intraoperative hemorrhage, however, no statistical significance in these factors was reported in our study. To our knowledge, thickness of neck might influence the operative time due to the difficulty of surgical exposure. Although surgical time tend to be longer in patients with thick neck, this trend did not reach statistical significance in our study.

Operational level was a vital factor affecting the difficulty of ACSS. In our study, as operational segments increased, prolonged operative time and more intraoperative blood loss were reported and these findings were consistent with previous research [16]. Moreover, the incidence of complications associated with the surgical procedure increased when more segments were decompressed and fused. Kou et al. [17] reported that multilevel procedures had a significantly higher risk for epidural hematoma. Sagi et al. [1] also reported that the number of vertebral bodies exposed more than three levels and operative time longer than $5 \mathrm{~h}$ were statistically associated with increased risk for airway complications with potentially catastrophic consequences. The selection of operational levels is mainly based on detailed clinical evaluation including extent of compression, chief complaint and the patient's general condition. Adequate neurological decompression is essential for optimal clinical outcomes but caution should be made to reduce the affected segments to minimize surgical trauma and risk of potential complications.

The study demonstrated that OPLL was another predictive factor for high-difficulty surgery. The technical challenges faced when treating OPLL depend on the decompression procedure including less injury to the spinal cord and better protection of the venous plexus especially when combining dural ossification [18]. Furthermore, OPLL is associated with high risks of perioperative complications, such as cerebrospinal fluid leakage, dysphonia, dysphagia and neurological impairment [19]. 
Spinal canal occupational ratio was used to represent the degree of compression and high signal intensity of spinal cord on T2-weighted images closely correlated with severe spinal cord compression [20]. This study confirmed that the spinal canal occupational ratio and high signal intensity were risk factors for surgical difficulty. Therefore, surgeons should be cautious when severe compression and high signal intensity are observed on MR images.

For the clinical use of the established nomogram, a $0.906 \mathrm{C}$-index was reported for the model in addition to a good calibration curve for use in predicting the probability of high-difficulty surgery.

There are several limitations of this study that need to be addressed. First, this is a retrospective study. Second, this study was conducted in a single institution and the limited number of cases might restrict the value of its clinical use. Due to these limitations, prospective multicenter studies with a large sample size are needed to validate our results.

\section{Conclusions}

This study indicated that the number of operational levels, OPLL, high signal intensity of spinal cord, and spinal canal occupational ratio were independently associated with surgical difficulty and a predictive nomogram can be established using the identified risk factors. Optimal performance was achieved for predicting surgical difficulty of ACSS based on preoperative factors.

\section{Abbreviations}

ACSS: Anterior cervical spine surgery; OPLL: Ossified posterior longitudinal ligament; PACS: Picture archiving and communication system; ROC: Receiver operating characteristics (ROC) curve; OR: Odds ratio.

\section{Acknowledgements}

Not applicable.

\section{Authors' contributions}

CYJ, YLR, SZY designed this study. JXW performed radiographic analysis. GYY, JF performed review of electronic patient record. WHC performed surgeries. CYJ, PYT performed data analysis and interpretation. CYJ, DDJ, WL, XHG prepared the manuscript, tables, and figures. All authors have read and approved the final manuscript.

\section{Funding}

This work was sponsored by the Natural Science Foundation of Jiangsu (Grant No. BK20181490), Wu Jieping Medical Foundation (Grant No: 320-2745-16117), the Six Talent Peaks Project in Jiangsu Province (Grant No. TD-SWYY-010) and the National Natural Science Foundation of China (Grant No. 81974335). The funding bodies were not involved in the conduct of the research, or in the data analysis and interpretation of the manuscript.

\section{Availability of data and materials}

The data are not publicly available due the privacy of patients included but are available from the corresponding author on reasonable request for academic research purpose.

\section{Ethics approval and consent to participate}

Our retrospective study was approved by the Ethics Committee of The First Affiliated Hospital of Nanjing Medical University and written informed consent was obtained from all participants.

\section{Consent for publication}

Not applicable.

\section{Competing interests}

The authors declare that they have no competing interests.

Received: 27 July 2020 Accepted: 20 December 2020

Published online: 29 March 2021

\section{References}

1. Sagi HC, Beutler W, Carroll E, Connolly PJ. Airway complications associated with surgery on the anterior cervical spine. Spine. 2002;27:949-53. https://doi.org/10.1097/00007632-200205010-00013.

2. Mayo BC, Massel DH, Bohl DD, Long WW, Modi KD, Singh K. Anterior cervical discectomy and fusion: the surgical learning curve. Spine. 2016;41:1580-5. https://doi.org/10.1097/brs.0000000000001588.

3. Fountas KN, Kapsalaki EZ, Nikolakakos LG, Smisson HF, Johnston KW, Grigorian AA, Lee GP, Robinson JS Jr. Anterior cervical discectomy and fusion associated complications. Spine. 2007;32:2310-7. https://doi.org/ 10.1097/BRS.0b013e318154c57e.

4. Nanda A, Sharma M, Sonig A, Ambekar S, Bollam P. Surgical complications of anterior cervical diskectomy and fusion for cervical degenerative disk disease: a single surgeon's experience of 1,576 patients. World Neurosurg. 2014;82(6):1380-7.

5. Zhang Y, Liu H, Yang H, Pi B. Anterior cervical corpectomy and fusion versus discectomy and fusion for the treatment of two-level cervical spondylotic myelopathy: analysis of sagittal balance and axial symptoms. Int Orthop. 2018;42:1877-82. https://doi.org/10.1007/s00264-018-3804-3.

6. Nurjck S. The pathogenesis of the spinal cord disorder associated with cervical spondylosis. Brain. 1972;95:87-100. https://doi.org/10.1093/ brain/95.1.87.

7. Badhiwala JH, Wilson JR. The natural history of degenerative cervical myelopathy. Neurosurg Clin N Am. 2018;29:21-32.

8. Patil PG, Turner DA, Pietrobon R. National trends in surgical procedures for degenerative cervical spine disease: 1990-2000. Neurosurgery. 2005;57:753-8.

9. Fessler RG, Steck JC, Giovanini MA. Anterior cervical corpectomy for cervical spondylotic myelopathy. Neurosurgery. 1998;43:257-65.

10. Mazas S, Benzakour A, Castelain JE, Damade C, Ghailane S, Gille O. Cervical disc herniation: which surgery? Int Orthop. 2019;43:761-6. https://doi. org/10.1007/s00264-018-4221-3.

11. Natkaniec M, Dworak J, Pedziwiatr M, Pisarska M, Major P, Dembinski M, Winiarski M, Budzynski A. Patients criteria determining difficulty of the laparoscopic lateral transperitoneal adrenalectomy. A retrospective cohort study. Int J Surg (London, England). 2017;43:33-7. https://doi.org/ 10.1016/j.jisu.2017.05.032.

12. Hasegawa Y, Wakabayashi G, Nitta H, Takahara T, Katagiri H, Umemura A, Makabe K, Sasaki A. A novel model for prediction of pure laparoscopic liver resection surgical difficulty. Surg Endosc. 2017;31:5356-63. https:// doi.org/10.1007/s00464-017-5616-8.

13. D'Orazi V, Sacconi A, Trombetta S, Karpathiotakis M, Pichelli D, Di Lorenzo E, Ortensi A, Urciuoli P, Biffoni M, Ortensi A. May predictors of difficulty in thyroid surgery increase the incidence of complications? Prospective study with the proposal of a preoperative score. BMC Surg. 2019;18:116. https://doi.org/10.1186/s12893-018-0447-7.

14. Liu Z, Gong C, Liu Y, Zhang L. Establishment of a scoring system for predicting the difficulty level of high-intensity focussed ultrasound ablation of uterine fibroids. Int J hyperthermia. 2018;34:77-86. https://doi.org/10. 1080/02656736.2017.1325015.

15. Owens RK 2nd, Djurasovic M, Onyekwelu I, Bratcher KR, McGraw KE, Carreon LY. Outcomes and revision rates in normal, overweight, and obese patients 5 years after lumbar fusion. Spine J. 2016;16:1178-83. https://doi.org/10.1016/j.spinee.2016.06.005. 
16. Yu S, Li F, Yan N, Yuan C, He S, Hou T. Anterior fusion technique for multilevel cervical spondylotic myelopathy: a retrospective analysis of surgical outcome of patients with different number of levels fused. PLoS ONE. 2014;9:e91329. https://doi.org/10.1371/journal.pone.0091329.

17. Kou J, Fischgrund J, Biddinger A, Herkowitz H. Risk factors for spinal epidural hematoma after spinal surgery. Spine. 2002;27:1670-3. https:// doi.org/10.1097/00007632-200208010-00016.

18. Chen Y, Guo Y, Lu X, Chen D, Song D, Shi J, Yuan W. Surgical strategy for multilevel severe ossification of posterior longitudinal ligament in the cervical spine. J Spinal Disord Tech. 2011;24:24-30. https://doi.org/10. 1097/BSD.0b013e3181c7e91e.

19. Morishita S, Yoshii T, Okawa A, Fushimi K, Fujiwara T. Perioperative complications of anterior decompression with fusion versus laminoplasty for the treatment of cervical ossification of the posterior longitudinal ligament: propensity score matching analysis using a nation-wide inpatient database. Spine J. 2019;19:610-6. https://doi.org/10.1016/j.spinee.2018. 10.013 .

20. Takahashi M, Yamashita Y, Sakamoto Y, Kojima R. Chronic cervical cord compression: clinical significance of increased signal intensity on MR images. Radiology. 1989;173:219-24. https://doi.org/10.1148/radiology. 173.1.2781011.

\section{Publisher's Note}

Springer Nature remains neutral with regard to jurisdictional claims in published maps and institutional affiliations.
Ready to submit your research? Choose BMC and benefit from:

- fast, convenient online submission

- thorough peer review by experienced researchers in your field

- rapid publication on acceptance

- support for research data, including large and complex data types

- gold Open Access which fosters wider collaboration and increased citations

- maximum visibility for your research: over 100M website views per year

At BMC, research is always in progress.

Learn more biomedcentral.com/submissions 